\title{
Classificação da composição iônica da água de irrigação usando regressão linear múltipla
}

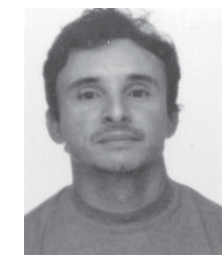

Celsemy E. Maia ${ }^{1}$ Elís R.C. de Morais² \& Maurício de Oliveira ${ }^{3}$

${ }^{1}$ ESAM/DPS. R. Miro F. de Mendonça, 130, Planalto 13 de Maio, CEP 59633-010, Mossoró, RN. E-mail: celsemymaia@bol.com.br (Foto)

2 AGROMASTER

3 ESAM/DPS. E-mail: mauricio@esam.br

Protocolo $087-11 / 07 / 2000$

\begin{abstract}
Resumo: Objetivou-se, com o presente trabalho, desenvolver uma metodologia para classificação da composição iônica da água de irrigação, através da regressão linear múltipla, tendo-se, como variável dependente, a condutividade elétrica e, como variáveis independentes, as concentrações de cátions e ânions da água de irrigação, classificada de acordo com o peso de cada íon no modelo estatístico. A fonte secundária de dados para a pesquisa foi o Banco de Dados do Laboratório de Análise de Água e Fertilidade do Solo, da Escola Superior de Agricultura de Mossoró (LAAFS/ESAM). As regressões foram ajustadas utilizando-se o método da seleção por etapas, conhecido como the stepwise regression procedure, no qual a variável dependente foi a condutividade elétrica e, como variáveis independentes, os íons determinados pela análise físico-química da água. Os resultados mostraram que, empregando-se este critério de regressão linear múltipla, havia variação na contribuição de cada variável no modelo ajustado, cuja estimativa era baseada no aumento da soma de quadrado, devido à regressão, a medida em que se incorporava, ao modelo, cada variável independente. Em função de critérios preestabelecidos, águas provenientes de mananciais da região da Chapada do Apodi foram classificadas como cálcica-sódica, cálcica e cloretada, quando provinham de poço tubular, de poço amazonas e rio, respectivamente. As águas oriundas da região do Baixo Açu, foram classificadas como sódica, magnesiana-sódica e sódica, para as águas de poço tubular, poço amazonas e rio, respectivamente.
\end{abstract}

Palavras-chave: classificação de águas, água de irrigação, regressão linear múltipla

\section{Classification of the ionic composition of the irrigation water using multiple linear regression}

\begin{abstract}
This work was conducted with the objective of developing a methodology for classification of the ionic composition of the irrigation water using multiple linear regression. A Stepwise Regression Analysis model was tested, using electrical conductivity as the dependent variable and analyzed ions calcium, sodium, potassium, carbonate, bicarbonate and chlorides as the independent variables in all tested models. All water samples were collected by the farmers of the region where this work was conducted. The regression models were adjusted using the water analysis database from the ESAM's Analysis Laboratory (Laboratório de Análises de Água e Fertilidade do Solo da Escola Superior de Agricultura de Mossoró - LAAFS/ESAM). The linear model, adjusted using the Stepwise Regression Procedure, shows that the degree of model adjustment tested depends upon geological formation of watersheds and whether it is collected in a river or tubular wells. The classification of the water in calcareous region of the Chapada do Apodi is calcic-sodic, calcic or choride if this source was tubular well, piezometric well (drilled in unconfined water denominated in the region as poço amazonas) or surface rivers and lagoons water, respectively. In Baixo Açu region, these waters were classified as sodic, magnesian-sodic or sodic depending if the source collected is a tubular well (drilled in Açu sedimentary geological formation), piezometric well or superficial water, respectivelly.
\end{abstract}

Key words: water classification, irrigation water, multiple linear regression

\section{INTRODUÇÃO}

A composição iônica da água é de suma importância quando se deseja avaliar a sua qualidade para fins agronômicos e, mais especificamente, para uso na irrigação. Existe uma grande variação nas classificações de água para fins de irrigação, embora haja um consenso de que o equilíbrio iônico e a salinidade da água sejam fatores decisivos para a avaliação da qualidade da água, para fins de irrigação. Shalhevet \& Kamburov (1976) efetuaram extensivo levantamento de diferentes esquemas de classificação usados em todo o mundo e verificaram que a maioria deles é baseada na razão de adsorção de sódio (RAS) e na condutividade elétrica (CE). Laboratórios de alguns países incluem boro em sua classificação de águas, para o mesmo fim; 
outros os associam às características e propriedades físicas e químicas dos solos em que as águas vão ser utilizadas. Doneen (1975) enfatiza a necessidade desta relação da água com a permeabilidade que a mesma poderá possuir em contato com o solo, classificando as águas em função do índice de permeabilidade (IP) que nada mais é do que o equilíbrio químico dado pela expressão:

$$
\mathrm{IP}=\left[\mathrm{Na}+\left(\mathrm{HCO}_{3}\right)^{0,5}\right] /(\mathrm{Ca}+\mathrm{Mg}+\mathrm{Na})
$$

Algumas classificações, no entanto, possuem falhas, visto que, ao se referirem à composição da água, baseiam-se exclusivamente no equilíbrio entre cátions, sintetizados na RAS, enquanto outras se fundamentam como proporção dominante de cloreto nos ânions em solução e, algumas, quando se referem a ânions, empregam apenas bicarbonato (Audry \& Suassuna, 1995) à semelhança da classificação proposta por Doneen (1975).

Davis \& DeWiest (1966) sugerem um esquema de classificação com um diagrama apresentando a composição iônica da água de irrigação em escala percentual, em função das concentrações de cálcio, magnésio e da soma de sódio e potássio e, ainda, pelos ânions cloretos, sulfatos e soma dos carbonatos com bicarbonatos. Os autores apresentam esta classificação de água como uma das mais rigorosas, porém com uma restrição, posto que nem sempre os laboratórios de análises de rotina expressam todos os cátions e ânions necessários para se obter a distribuição percentual (\%) obtida a partir dos resultados analíticos expressos em $\mathrm{mmol}_{\mathrm{c}} \mathrm{L}^{-1}$. Esta representação consiste em dois triângulos, um para os cátions e outro para os ânions. Um losango se encaixa entre os dois triângulos, utilizando os mesmos eixos e as mesmas escalas que os lados dos triângulos imediatamente paralelos, ou seja, $\mathrm{Na}^{+}$e $\mathrm{K}^{+}$de um lado e $\mathrm{HCO}_{3}^{-}+$ $\mathrm{CO}_{3}=$ de outro. Os lados opostos do triângulo correspondem aos grupos de íons complementares, ou seja, $\mathrm{Ca}^{++}+\mathrm{Mg}^{++}$de um lado e $\mathrm{SO}_{4}=$ e $\mathrm{Cl}^{-}$do outro lado; assim, o "Triângulo de Piper" explicita os termos utilizados para exprimir, respectivamente para cátions e ânions, a dominância de tal ou qual íon ou grupo de íons, e mostra no losango como são combinados esses qualificativos para denominar uma água, por exemplo, de bicarbonatada-cálcica, cloretada-sódica etc.

A condutividade elétrica (CE) é a determinação mais empregada para expressar a concentração de sais solúveis na água de irrigação. Esta medida é o inverso da resistividade elétrica e corresponde à medida da capacidade de uma água em conduzir eletricidade, crescendo proporcionalmente a medida em que a concentração de sais aumenta (Holanda \& Amorim, 1997; Ferreira, 1997). Assim, os Sólidos Dissolvidos Totais (SDT) podem ser estimados pela condutividade elétrica, pela relação: SDT $\left(\mathrm{mg} \mathrm{L}^{-1}\right)=640 * \mathrm{CE}\left(\mathrm{dS} \mathrm{m}^{-1}\right)$ (Holanda \& Amorim, 1997) porém a multiplicação da CE por 640 só é recomendada para águas com CE inferior a $5 \mathrm{dS} \mathrm{m}^{-1}$. Nicolás et al. (1998) encontraram valores diferentes para este fator, que multiplica a CE, sendo utilizados 780 ou 900, para águas cujo ânion predominante seja o cloreto ou o sulfato, respectivamente.

Atualmente, a regressão linear múltipla é uma técnica largamente empregada por todos aqueles que se dedicam à pesquisa, mas ela deve ser empregada apenas em circunstâncias bem definidas, para que o modelo estatístico utilizado represente bem o fenômeno em estudo analisado, pois somente se conhecendo a verdadeira relação entre as variáveis dependentes e as variáveis independentes, pode-se fazer inferências estatísticas precisas com utilidades práticas para controlar e predizer as respostas para seu estudo, como preconizam também Regazzi \& Leite (1992).

O objetivo deste trabalho foi desenvolver uma metodologia para classificação da composição iônica da água de irrigação, usando-se a regressão linear múltipla, e se tendo, como variável dependente, a condutividade elétrica e, independentes, as concentrações de cátions e ânions da água de irrigação, classificada de acordo com o peso de cada íon no modelo estatístico.

\section{MATERIAL E MÉTODOS}

Os dados utilizados para a presente pesquisa foram provenientes do banco de dados gerados para emissão dos boletins de análises de água, emitidos pelo Laboratório de Análises de Água e Fertilidade do Solo da Escola Superior de Agricultura de Mossoró, RN (LAAFS/ESAM) entre os anos de 1990-1995, num total de 607 amostras analisadas. O banco de dados foi estruturado em xBase e analisado empregando-se o software SCASA (Maia \& Morais, 1996). As análises de regressão linear múltipla foram efetuadas através do software SAEG, desenvolvido pela Universidade Federal de Viçosa, MG. Do volume total de amostras de água que chegam para análise no LAAFS/ESAM, mais de $80 \%$ provêm da região comercialmente denominada pólo hortifrutigranjeiro, do Estado do Rio Grande do Norte, inserida na mesorregião 18 (sertão semi-árido) e nas microrregiões 79 (Salineira Norte-Riograndense) e 81 (Açu e Apodi) conforme IBGE (1974) citado em Brasil (1978). Daí a opção, quanto ao maior detalhamento do estudo, nas águas dessas duas microrregiões, na presente pesquisa.

\section{Fisiografia da área de abrangência do estudo}

A microrregião salineira norte-rio-grandense, é composta, atualmente, por oito municípios (Alto do Rodrigues, Areia Branca, Carnaubais, Grossos, Guamaré, Macau, Mossoró e Pendências) enquanto a microrregião Açu e Apodi é constituída de 15 municípios (Açu, Apodi, Campo Grande, Caraúbas, Felipe Guerra, Governador Dix-Sept Rosado Ipanguaçu, Itajá, Itaú, Janduís, Paraú, São Rafael, Serra do Mel, Severiano Melo e Upanema). Comercialmente, no entanto, as regiões de maior expressividade agrícola são denominadas Baixo Açu (que compreendem a região e enclaves da planície aluvional do baixo curso do Rio Piranhas-Açu) liderada pela cidade do Assu e pela Chapada do Apodi, liderada comercialmente, por sua vez, pela cidade de Mossoró.

As áreas supra mencionadas se localizam no extremo noroeste do Estado do Rio Grande do Norte, na quadrícula geográfica entre os paralelos $4^{\circ} 48^{\prime}$ a $5^{\circ} 41^{\prime}$ de latitude Sul e os meridianos $37^{\circ} 30^{\prime}$ a $38^{\circ} 5^{\prime}$ a Oeste de Greenwich. Em ambas as áreas estudadas predomina o tipo bioclimático de Gausen 4aTh - Tropical quente de seca acentuada, com índice xerotérmico entre 150 e 200 . Pela classificação de Thornthwaite, baseada numa série de índices térmicos utilizando-se o balanço hídrico, as regiões apresentam clima do tipo DdA'a', ou seja, semi-árido megatérmico, com pouco ou nenhum excesso de água durante o ano. Pelo regime térmico e pluviométrico, as regiões apresentam 
clima do tipo BSw’h', segundo Köppen, isto é, clima semi-árido, onde a estação chuvosa atrasa para o outono, sendo a maior incidência de chuvas do verão para o outono. As chuvas têm distribuição bastante irregular no tempo e no espaço, aumentando sobremaneira o risco climático. A média anual de precipitação é de aproximadamente $680 \mathrm{~mm}$. Devido à baixa latitude e à ausência de fatores geográficos influenciadores, a temperatura apresenta-se sem grande variação anual. A umidade relativa é predominantemente baixa em toda a região. Estação climatológica inserida na região do estudo (http://www.esam.br/ servicos) evidencia uma média anual de temperatura de aproximadamente $27,5^{\circ} \mathrm{C}$, sendo que o mês mais quente é dezembro, com média de $28,5^{\circ} \mathrm{C}$, e o mês mais frio é julho, com média de $26,5^{\circ} \mathrm{C}$. As temperaturas máxima e mínima do ar têm valores médios iguais a 33,3 e $22,7^{\circ} \mathrm{C}$, respectivamente (Carmo Filho et al, 1991).

\section{Análise química da água}

Nas análises de água realizadas pelo LAAFS/ESAM, são determinadas as seguintes características físico-químicas: $\mathrm{pH}$, $\mathrm{CE}$, cátions $\left(\mathrm{Ca}^{2+}, \mathrm{Mg}^{2+}, \mathrm{Na}^{+}\right.$e $\left.\mathrm{K}^{+}\right)$e ânions $\left(\mathrm{Cl}^{-}, \mathrm{HCO}_{3}{ }^{-}\right.$e $\left.\mathrm{CO}_{3}{ }^{2-}\right)$. É utilizada, para determinação dessas características, a metodologia proposta por Richards (1954). $\mathrm{Ca}^{2+}, \mathrm{Mg}^{2+}, \mathrm{Cl}^{-}, \mathrm{HCO}_{3}^{-}$ $\mathrm{e} \mathrm{CO}_{3}{ }^{2-}$ são determinados por volumetria de complexação e $\mathrm{Na}^{+} \mathrm{e}$ $\mathrm{K}^{+}$por fotometria de emissão de chama, com leituras simultâneas.

\section{Classificação da composição iônica da água de irrigação}

Para classificar a água de irrigação pela análise de regressão múltipla, utilizou-se a seleção por etapa (the stepwise regression procedure) de acordo com Draper \& Smith (1981) em que a variável dependente foi a condutividade elétrica e as variáveis independentes os íons determinados pela análise química da água $\left(\mathrm{Ca}^{++}, \mathrm{Mg}^{++}, \mathrm{Na}^{+}, \mathrm{K}^{+}, \mathrm{Cl}^{-}, \mathrm{HCO}_{3}^{-} \mathrm{e} \mathrm{CO}_{3}{ }^{=}\right)$.

Como critério para classificação da água (sódica, cálcica, magnesiana etc.) permaneciam no modelo obtido pelo ajuste da regressão múltipla, as variáveis independentes que melhor se ajustaram ao fenômeno, não apenas pelo critério da significância estatística mas, também, com base na contribuição de cada variável no modelo. Esta contribuição quantitativa foi estimada pelo aumento observado na soma de quadrado devido à regressão, quando da adição de cada variável no modelo. Por exemplo, considerando-se o modelo I igual a $\mathrm{Y}=\mathrm{a}+\mathrm{bx}_{1}+\mathrm{cx}_{2}+\mathrm{e}_{\mathrm{i}}$ e o modelo II igual a $\mathrm{Y}=\mathrm{a}+\mathrm{bx}_{1}+\mathrm{cx}_{2}+\mathrm{dx}_{3}+\mathrm{e}_{\mathrm{i}}$, calculada a diferença entre a soma de quadrado em virtude da regressão (SQreg) do modelo I e II e se fazendo a subtração da SQreg de II menos a SQreg de I, encontra-se o aumento na SQreg devido à adição no modelo da variável $\mathrm{x}_{3}$.

Somando-se todos os pesos das variáveis e o assumindo iguais a $100 \%$, faz-se a proporção percentual de cada variável. O maior peso, multiplicado por 0,9 , foi o valor limite para que os íons entrassem na classificação da água.

\section{RESULTADOS E DISCUSSÃO}

Pelos modelos de regressão linear propostos para as análises de água, foi possível identificar-se diferentes tipos de água quanto à sua composição iônica para as regiões da Chapada do Apodi e Baixo Açu (Tabela 1).

Pelos resultados obtidos para a região da Chapada do Apodi, observou-se que, utilizando-se todas as análises, independente da sua origem, o maior peso na condutividade elétrica no modelo ajustado é devido ao cálcio, seguido pelo sódio, cloreto e magnésio. O mesmo comportamento foi verificado para as análises de poço tubular. Para as águas de poço amazonas, observa-se que o potássio, mesmo contribuindo com menor peso, faz parte do modelo, o que não foi verificado para as águas de poço tubular e rio. Para as águas de rio, observa-se maior peso do cloreto, seguido do sódio, magnésio e bicarbonato, caso em que as águas de poço tubular e amazonas seriam classificadas como cálcicas, enquanto as águas de rio, como cloretadas.

Levando-se em consideração apenas o peso da soma de quadrados, para a região do Baixo Açu, observa-se que as águas de poço tubular e de rio são classificadas como sódicas, enquanto as de poço amazonas o são como magnesianas. A classificação magnesiana para as águas de poço amazonas, contraria Audry \& Suassuna (1995) que afirmam que não existe esta classificação para água de irrigação, porém ela pode ser devido ao fato dos autores levarem em consideração apenas as concentrações do elemento, obtidas quimicamente na água, não se levando em consideração seu efeito sobre os demais íons. Assim, se se levar em conta apenas os teores de cada íon, as águas serão classificadas apenas pela quantidade química e

Tabela 1. Equações de regressão linear múltipla calculadas pelo método Stepwise, coeficiente de determinação e contribuição de cada variável independente no modelo para as regiões da Chapada do Apodi e Baixo Açu

\begin{tabular}{|c|c|c|c|}
\hline Origem & Equação & $\mathrm{R}^{2}$ & Contribuição \\
\hline \multicolumn{4}{|c|}{ Chapada do Apodi } \\
\hline Todas & $\mathrm{CE}=0,1788+0,0378 \mathrm{Cl}+0,069 \mathrm{Mg}+0,0958 \mathrm{Ca}+0,067 \mathrm{Na}$ & 0,9839 & $\mathrm{Ca}>\mathrm{Na}>\mathrm{Cl}>\mathrm{Mg}$ \\
\hline Tubular & $\mathrm{CE}=0,1608+0,0337 \mathrm{Cl}+0,0715 \mathrm{Mg}+0,0998 \mathrm{Ca}+0,071 \mathrm{Na}$ & 0,9868 & $\mathrm{Ca}>\mathrm{Na}>\mathrm{Cl}>\mathrm{Mg}$ \\
\hline Rio & $\mathrm{CE}=0,1718+0,0675 \mathrm{Cl}+0,0579 \mathrm{Mg}+0,0476 \mathrm{Na}+0,0779 \mathrm{HCO}_{3}$ & 0,9680 & $\mathrm{Cl}>\mathrm{Na}>\mathrm{Mg}>\mathrm{HCO}_{3}$ \\
\hline \multicolumn{4}{|c|}{ Baixo Açu } \\
\hline Tubular & $\mathrm{CE}=-0,002+0,1161 \mathrm{Na}+0,0912 \mathrm{Mg}+0,2433 \mathrm{~K}+0,0737 \mathrm{Ca}$ & 0,9990 & $\mathrm{Na}>\mathrm{Mg}>\mathrm{K}>\mathrm{Ca}$ \\
\hline Amazonas & $\begin{aligned} \mathrm{CE}= & -0,0672+0,0443 \mathrm{Cl}+0,0694 \mathrm{HCO}_{3}+0,1198 \mathrm{Mg}+ \\
& 0,0648 \mathrm{Ca}+0,059 \mathrm{Na}\end{aligned}$ & 0,9891 & $\mathrm{Mg}>\mathrm{Ca}>\mathrm{HCO}_{3}>\mathrm{Na}>\mathrm{Cl}$ \\
\hline Rio & $\mathrm{CE}=0,1356+0,1527 \mathrm{Na}$ & 0,9985 & $\mathrm{Na}$ \\
\hline
\end{tabular}


não pela interação de um íon específico com os demais. Segundo Regazzi \& Leite (1992) é sempre importante o conhecimento químico, físico e biológico do problema estudado, caso em que a dependência da condutividade elétrica em função dos íons é algo de conhecimento amplo para aqueles que trabalham com qualidade de água, para fins de irrigação.

Observa-se, ainda, que para as água de rio do Baixo Açu, apenas o sódio explica a variação da condutividade elétrica com significativo coeficiente de determinação, demonstrando que, mesmo os outros íons entrando no modelo, não se observou incremento significativo no coeficiente de determinação, sendo que apenas o sódio explica a condutividade elétrica para essas águas; neste caso, poder-se-á estimar o teor de sódio pela condutividade elétrica mas, como é demonstrado na Tabela 2, o peso na soma de quadrado possui valores absolutos diferentes para cada local e origem das águas, sendo estes valores superiores nas águas da região da Chapada do Apodi; assim, assumindo-se o somatório dos pesos igual a $100 \%$, faz-se a porcentagem equivalente para cada peso.

Tabela 2. Peso na soma de quadrado e peso em porcentagem das variáveis de cada modelo ajustado pela regressão linear múltipla para as regiões da Chapada do Apodi e Baixo Açu

\begin{tabular}{|c|c|c|c|}
\hline Origem & Íon & $\begin{array}{l}\text { Peso na Soma } \\
\text { de Quadrado }\end{array}$ & $\begin{array}{c}\text { Peso em } \\
\text { Porcentagem }\end{array}$ \\
\hline \multicolumn{4}{|c|}{ Chapada do Apodi } \\
\hline \multirow{4}{*}{ Todas as análises } & $\mathrm{Ca}^{2+}$ & 53,15 & 36,21 \\
\hline & $\mathrm{Na}^{+}$ & 48,58 & 33,09 \\
\hline & $\mathrm{Cl}^{-}$ & 26,50 & 18,05 \\
\hline & $\mathrm{Mg}^{2+}$ & 18,57 & 12,65 \\
\hline \multirow{4}{*}{ Poço tubular } & $\mathrm{Ca}^{2+}$ & 40,99 & 37,64 \\
\hline & $\mathrm{Na}^{+}$ & 37,42 & 34,36 \\
\hline & $\mathrm{Cl}^{-}$ & 15,32 & 14,07 \\
\hline & $\mathrm{Mg}^{2+}$ & 15,17 & 13,93 \\
\hline \multirow{5}{*}{ Poço amazonas } & $\mathrm{Ca}^{2+}$ & 10,75 & 49,36 \\
\hline & $\mathrm{Na}^{+}$ & 5,12 & 23,51 \\
\hline & $\mathrm{Mg}^{2+}$ & 4,57 & 20,98 \\
\hline & $\mathrm{Cl}^{-}$ & 1,07 & 4,91 \\
\hline & $\mathrm{K}^{+}$ & 0,27 & 1,24 \\
\hline \multirow{4}{*}{ Rio } & $\mathrm{Cl}^{-}$ & 6,64 & 59,66 \\
\hline & $\mathrm{Na}^{+}$ & 2,46 & 22,10 \\
\hline & $\mathrm{Mg}^{2+}$ & 1,50 & 13,48 \\
\hline & $\mathrm{HCO}_{3}^{-}$ & 0,53 & 4,76 \\
\hline \multicolumn{4}{|c|}{ Baixo Açu } \\
\hline \multirow{7}{*}{ Todas as análises } & $\mathrm{Ca}^{2+}$ & 1,21 & 40,07 \\
\hline & $\mathrm{Na}^{+}$ & 0,86 & 28,48 \\
\hline & $\mathrm{Cl}^{-}$ & 0,29 & 9,60 \\
\hline & $\mathrm{K}^{+}$ & 0,25 & 8,28 \\
\hline & $\mathrm{Mg}^{2+}$ & 0,24 & 7,95 \\
\hline & $\mathrm{HCO}_{3}^{-}$ & 0,13 & 4,30 \\
\hline & $\mathrm{CO}_{3}{ }^{=}$ & 0,04 & 1,32 \\
\hline \multirow{4}{*}{ Poço tubular } & $\mathrm{Na}^{+}$ & 5,81 & 90,50 \\
\hline & $\mathrm{Mg}^{2+}$ & 0,27 & 4,21 \\
\hline & $\mathrm{K}^{+}$ & 0,25 & 3,89 \\
\hline & $\mathrm{Ca}^{2+}$ & 0,09 & 1,40 \\
\hline \multirow{5}{*}{ Poço amazonas } & $\mathrm{Mg}^{2+}$ & 0,27 & 23,48 \\
\hline & $\mathrm{Ca}^{2+}$ & 0,25 & 21,74 \\
\hline & $\mathrm{HCO}_{3}^{-}$ & 0,24 & 20,87 \\
\hline & $\mathrm{Na}^{+}$ & 0,22 & 19,13 \\
\hline & $\mathrm{Cl}^{-}$ & 0,17 & 14,78 \\
\hline
\end{tabular}

\begin{tabular}{|c|c|c|c|}
\hline $\operatorname{Rio}^{(*)}$ & $\mathrm{Na}^{+}$ & - & - \\
\hline
\end{tabular}
dessas águas
Neste sentido, o uso do peso em porcentagem levaria a uma precisão maior no diagnóstico, pois todos os elementos envolvidos nos modelos para cada local ou origem, estariam na mesma escala, no caso, expressos em valores relativos (percentagem).

Para classificação da água, encontrou-se o valor limite (VL) para se saber que elementos entrariam na classificação da água, entrando no modelo primeiro os íons de maior peso na soma de quadrado. $\mathrm{O}$ valor limite é calculado por $\mathrm{VL}=$ maior porcentagem $\mathrm{x} 0,9$.

Para as águas da região da Chapada do Apodi observa-se, para a regressão feita com todas as análises, que as águas foram classificadas como cálcica-sódica, pois se estimou o limite $\mathrm{VL}=32,59(36,21 \times 0,9)$ entrando na classificação o cálcio e o sódio, pois são valores, em porcentagem, superiores a VL. As demais classificações estão na Tabela 3 .

Tabela 3. Classificação da água pela análise da regressão linear múltipla para as regiões da Chapada do Apodi e Baixo Açu

\begin{tabular}{|c|c|}
\hline Origem & Classificação \\
\hline \multicolumn{2}{|c|}{ Chapada do Apodi } \\
\hline Todas as análises & Cálcica-sódica \\
\hline Poço tubular & Cálcica-sódica \\
\hline Poço amazonas & Cálcica \\
\hline Rio & Cloretada \\
\hline \multicolumn{2}{|c|}{ Baixo Açu } \\
\hline Todas as análises & Cálcica \\
\hline Poço tubular & Sódica \\
\hline Poço amazonas & Magnesiana-cálcica \\
\hline Rio & Sódica \\
\hline
\end{tabular}

A classificação proposta com base na regressão linear múltipla, mostra o efeito de cada íon na condutividade elétrica da água, podendo-se dizer, com base na força iônica da mesma, que existe uma relação positiva entre a condutividade elétrica e a força iônica; assim, a metodologia não leva em consideração os teores dos íons da análise química da água de irrigação, mas a interação provável entre eles. Na Tabela 4 verifica-se que, se levasse em consideração apenas o teor dos íons analisados, a

Tabela 4. Teores médios dos íons da água de irrigação para as regiões da Chapada do Apodi e Baixo Açu

\begin{tabular}{ccrr}
\hline Íons $^{(*)}$ & Tubular & \multicolumn{1}{c}{ Amazonas } & \multicolumn{1}{c}{ Rio } \\
\hline \multicolumn{4}{c}{ Chapada do Apodi } \\
\hline $\mathrm{Ca}^{2+}$ & 7,85 & 4,89 & 4,59 \\
$\mathrm{Mg}^{2+}$ & 6,55 & 4,48 & 5,07 \\
$\mathrm{~K}^{+}$ & 0,42 & 0,44 & 0,22 \\
$\mathrm{Na}^{+}$ & 10,68 & 9,32 & 8,74 \\
$\mathrm{Cl}^{-}$ & 17,12 & 13,77 & 12,65 \\
$\mathrm{HCO}^{-}$ & 4,79 & 3,30 & 3,16 \\
$\mathrm{CO}_{3}{ }^{-}$ & 0,36 & 0,24 & 0,40 \\
\hline \multicolumn{4}{c}{ Baixo Açu } \\
\hline $\mathrm{Ca}^{2+}$ & 2,34 \\
$\mathrm{Mg}^{2+}$ & 2,57 & 2,71 & 1,45 \\
$\mathrm{~K}^{+}$ & 0,48 & 0,29 & 1,37 \\
$\mathrm{Na}^{+}$ & 6,40 & 4,99 & 0,18 \\
$\mathrm{Cl}^{-}$ & 8,06 & 6,87 & 3,94 \\
$\mathrm{HCO}_{3}^{-}$ & 2,45 & 2,61 & 5,64 \\
$\mathrm{CO}_{3}{ }^{-}$ & 0,07 & 0,23 & 1,89 \\
& \multicolumn{3}{c}{0,17} \\
\hline
\end{tabular}

(9) Valores expressos em $\mathrm{mmol}^{-1}$ 
classificação seria diferente da proposta neste trabalho, porém definida de forma empírica.

Quando a classificação foi realizada utilizando-se todas as águas, independente da fonte, pode-se observar, para a região da Chapada do Apodi que as águas de rio diferiram completamente quando comparadas com as demais. Para o Baixo Açu, o efeito foi maior, ou seja, a classificação com todas as águas diferiu completamente da classificação realizada por fonte, mostrando que, estatisticamente é possível classificar-se águas, porém para uso prático o ideal é classificá-las por região e, dentro desta, para determinada fonte.

A diferença na classificação das águas para as duas regiões estudadas pode ser explicada principalmente pelos fatores edáficos e hidrogeológicos, que diferem para as duas regiões, onde a região da Chapada do Apodi apresenta solos derivados de calcário enquanto no Baixo Açu a predominância é de solos aluviais e solos derivados do arenito e cristalino. Na Chapada do Apodi as águas provêm de aqüíferos do calcário da formação Jandaíra, o que, na sua grande maioria, é influenciado pelo tipo de solo presente na região (Cambissolos Háplicos, Chernossolos, Vertissolos e Neossolos) que, indiretamente, também afetam a qualidade das águas superficiais que, por sua vez, drenam a bacia Potiguar nesse trecho de pedobiomas de domínios calcários. Segundo Santos et al. (1984) existe relação entre a qualidade da água com o tipo de solo da região, o que corrobora com a hipótese levantada; além disso, a classificação mostrada na Tabela 3 vai de encontro aos dados de Maia et al. (1997) que verificaram que a maioria das águas na região da Chapada do Apodi, principalmente de poço tubular, apresenta risco de precipitação de fosfato devido aos elevados teores de cálcio, verificado na prática para a região da Chapada do Apodi, principalmente para quem pratica a irrigação localizada por gotejamento. Para a região do Baixo Açu, Maia et al. (1998) observaram que as águas desta região não tinham predisposição para precipitação de $\mathrm{CaCO}_{3}$. Deve-se enfatizar, neste caso, que os poços tubulares e amazonas na Região do Baixo Açu, são influenciados diretamente pelo aqüífero Açu que, por sua vez, são influenciados pelas características dos Luvissolos Crômicos, Neossolos Quartzarênicos e, no caso dos Neossolos Flúvicos, há também influência da mineralogia dos materiais ao longo da bacia de drenagem que, em alguns casos, contribuem para aumentar a salinidade do solo e das águas (Souza et al., 1995). Em algumas amostras de água da bacia do Pataxós, por exemplo, há tendência de íons cloreto predominarem sobre os demais, apresentando valores absolutos muito elevados (Oliveira \& Resende, 1990).

\section{CONCLUSÕES}

1. O emprego da regressão linear múltipla permitiu classificar as águas com base na contribuição de cada variável no modelo ajustado.

2. As águas da Chapada do Apodi foram classificadas como cálcica-sódica, cálcica e cloretada para as fontes poço tubular, poço amazonas e rio, respectivamente.

3. As águas do Baixo Açu foram classificadas como sódica, magnesiana-sódica e sódica para as fontes poço tubular, poço amazonas e rio, respectivamente.

\section{LITERATURA CITADA}

Audry, P.; Suassuna, J.A. Salinidade das águas disponíveis para a pequena irrigação, no sertão nordestino. Recife: Fundação Joaquim Nabuco/CNPq, 1995, 128p.

Brasil, Ministério da Agricultura, Secretaria Nacional de Planejamento Agrícola. Aptidão das terras do Rio Grande do Norte. Brasília: BINAGRI, 1978, 84p,

Carmo Filho, F.; Espíndola Sobrinho, J.; Maia Neto, J.M. Dados meteorológicos de Mossoró (Jan de 1988 a Dez de 1990). Mossoró: ESAM/FGD, 1991, 121p. Coleção Mossoroense

Davis, S.N.; Dewiest, R.J.M. Hydrogeology. New York: John Wiley \& Sons, 1966, 463p.

Doneen, L.D. Water quality for irrigated agriculture. In: PoljakoffMayber, Gale, J. (ed). Plants in saline environments. New York: Springer-Verlag, 1975. p.56-76.

Draper, N.R.; Smith, H. Applied regression analysis. 2.ed. New York: John Wiley \& Sons, 1981, 709p.

Ferreira, P.A. Aspectos físico-químicos do solo, In: Gheyi, H.R.; Queiroz, J.E.; Medeiros, J.F. de. (ed). Manejo e controle da salinidade na agricultura irrigada. Campina Grande: UFPB, 1997, p.37-67.

Holanda, J.S. de; Amorim, J.R.A. de. Qualidade da água para irrigação, In: Gheyi, H.R.; Queiroz, J.E.; Medeiros, J.F. de. (ed). Manejo e controle da salinidade na agricultura irrigada. Campina Grande: UFPB, 1997, p.137-169.

Maia, C.E.; Morais, E.R.C. SCASA - Sistema de cadastramento de análises de solo e água. In: Congresso Latino Americano de Ciência do Solo, 13, 1996, Águas de Lindóia. Resumos... Piracicaba: SBCS, 1996. CD Rom.

Maia, C.E., Morais, E.R.C.; Oliveira, M. Qualidade da água para fertirrigação por gotejamento. I. Região da Chapada do Apodi, RN. Engenharia na Agricultura. Viçosa, MG, v.5, n.4. p.293-307. 1997

Maia, C.E.; Morais, E.R.C.; Oliveira, M. Qualidade da água para fertirrigação por gotejamento. II. Região do Baixo Açu, RN. Engenharia na Agricultura. Viçosa, MG, v.6, n.1. p.12-26. 1998

Nicolas, J.J.M.; Montesinos, M.G.; Tome, J.M.; Camara, M.A.O.; Camara, J.M.F. La calidad agronómica de los aguas de pozo en la Veja Baja del Segura durante los años de sequía. Revista Agrícola Vergel, Valencia, 1998. p.407.

Oliveira, M. de; Resende, M. Los suelos aluviales eutroficos (fluvents) de Baixo Açu y la adopción de agricultura de altos insumos: problemas y perspectivas. In. Congreso Latinoamericano de la Ciencia del Suelo, 9, La Habana, 11 17 de Marzo de 1990. 246p. Programa y Resúmenes

Regazzi, A.J.; Leite, H.G. Análise de regressão: Teoria à aplicação em manejo florestal. Viçosa, MG, 1992. 236p. Apostila

Richards, L.A. Diagnosis and improvement of saline and alkali soils. Washigton: USSL, 1954. 160p. USDA Handbook 60

Santos, J.P., Azevedo, S.G.; Mistretta, G. Novos aspectos da salinização das águas subterrâneas do cristalino do Rio Grande do Norte. São Paulo, SP: IPT, 1984.27p.

Shalhevet, J.; Kamburov, J. Irrigation and salinity: A world-wide survey. New Delhi: Caxton Press, 1976. 106p.

Sousa, A.R.; Batista, R.B.; Silva, A.B.; Oliveira; M. de.; Resende, M. Plagioclásios calco-sódicos de solos do Nordeste: Determinação por difração de raios X e implicações na sodificação. In. Congresso Brasileiro de Ciência do Solo, 25, 1995, Viçosa, MG. Resumos... Viçosa, MG: SBCS, v.1, p.271-273. 1995. 